\title{
Development of a Novel Quality Improvement Indicator Based on the Hemolysis Index
}

\author{
Eun Jin Lee, M.D., Miyoung Kim, M.D., Han-Sung Kim, M.D., Min-Jeong Park, M.D., Young Kyung Lee, M.D., \\ and Hee Jung Kang, M.D. \\ Department of Laboratory Medicine, Hallym University College of Medicine, Anyang, Korea
}

\begin{abstract}
Hemolysis frequently causes preanalytical errors in laboratory measurements. We aimed to develop a quality improvement indicator for evaluating the extent of inappropriate procedures causing hemolysis in clinical samples collected in medical care units. We defined the threshold value of the hemolysis index ( $\mathrm{H}$ index) causing significant interference with analyte measurement and analyzed the $\mathrm{H}$ index values of clinical samples in relation to the threshold. The $\mathrm{H}$ index threshold value causing a $10 \%$ bias in the measurement of lactate dehydrogenase was found to be 25 . The monthly mean $\mathrm{H}$ index and monthly frequency of samples with an $\mathrm{H}$ index $>25$ were significantly different among the types of ward $(P=0.001$, respectively), and significantly decreased after replacement of a laboratory centrifuge lacking temperature control $(20.6 \pm 0.58$ vs $23.30 \pm 1.08, P=0.01$; $23.4 \pm 1.69 \%$ vs $32.6 \pm 1.78 \%, P=0.01$ ). The monthly mean $\mathrm{H}$ index and the monthly frequency of samples with an $\mathrm{H}$ index above a threshold value may be useful quality improvement indicators for detection of inappropriate procedures in the acquisition and handling of blood samples in medical care units.
\end{abstract}

Key Words: $\mathrm{H}$ index, Hemolysis, Quality improvement indicator, Preanalytical error

\author{
Received: January 4, 2016 \\ Revision received: May 31, 2016 \\ Accepted: July 5, 2016

\begin{abstract}
Corresponding author: Hee Jung Kang Department of Laboratory Medicine, Hallym University College of Medicine, 22 Gwanpyeong-ro 170beon-gil, Dongan-gu, Anyang 14068, Korea Tel: +82-31-380-3929

Fax: +82-31-380-3934
\end{abstract} \\ E-mail: kangheejung@hallym.ac.kr
}

\begin{abstract}
() The Korean Society for Laboratory Medicine This is an Open Access article distributed under the terms of the Creative Commons Attribution Non-Commercial License (http://creativecommons.org/licenses/by-nc/4.0) which permits unrestricted non-commercial use, distribution, and reproduction in any medium, provided the original work is properly cited.
\end{abstract}

Hemoglobin interference is the most common cause of preanaIytical errors in clinical laboratories [1-7]. It is mainly caused by in vitro processes such as incorrect acquisition and handling procedures that cause hemolysis [8]. To prevent $\mathrm{Hb}$ interference-related errors, each sample should be examined visually immediately after centrifugation. However, this is not realistic considering the high number of samples that need to be processed at any given time in a clinical laboratory. To overcome the inherent limitations of visual examination, the hemolysis index ( $\mathrm{H}$ index) was developed to provide automated determination of potential interference in a sample using specific absorbance values $[2,3,6]$. The use of the $\mathrm{H}$ index for identification of error-prone samples is expected to improve the quality and efficiency of sample processing in clinical laboratories. In addition, it was reported that the frequency of the normal $\mathrm{H}$ index values of clinical samples from the outpatient department (OPD) is higher than that from the general ward (GW) or emergency room (ER) [9]. This finding suggests that the $\mathrm{H}$ index can be informative for assessing the correctness of the procedures for the acquisition and handling of blood samples in each type of a medical care unit. Thus, we aimed to develop a quality improvement indicator for detection of inappropriate procedures causing hemolysis in clinical samples using $\mathrm{H}$ index values of blood samples in a hospital.

$\mathrm{H}$ index results from the Hitachi MODULAR Analytics system (Roche Diagnostics, Basel, Switzerland) were verified according to the Clinical Laboratory and Standards Institute guidelines C56$\mathrm{A}$ [10] and revealed a linear relation with $\mathrm{Hb}$ concentration measured by spectrophotometry (UV-3600, SHIMADZU, Tokyo, Japan) (Fig. 1A). The lactate dehydrogenase (LD) assay, which is known to be strongly affected by hemolysis in a clinical sample [11], was chosen for the evaluation of interference related to hemolysis. For preparation of the test sample, whole blood from one healthy donor was divided into six aliquots, which were 

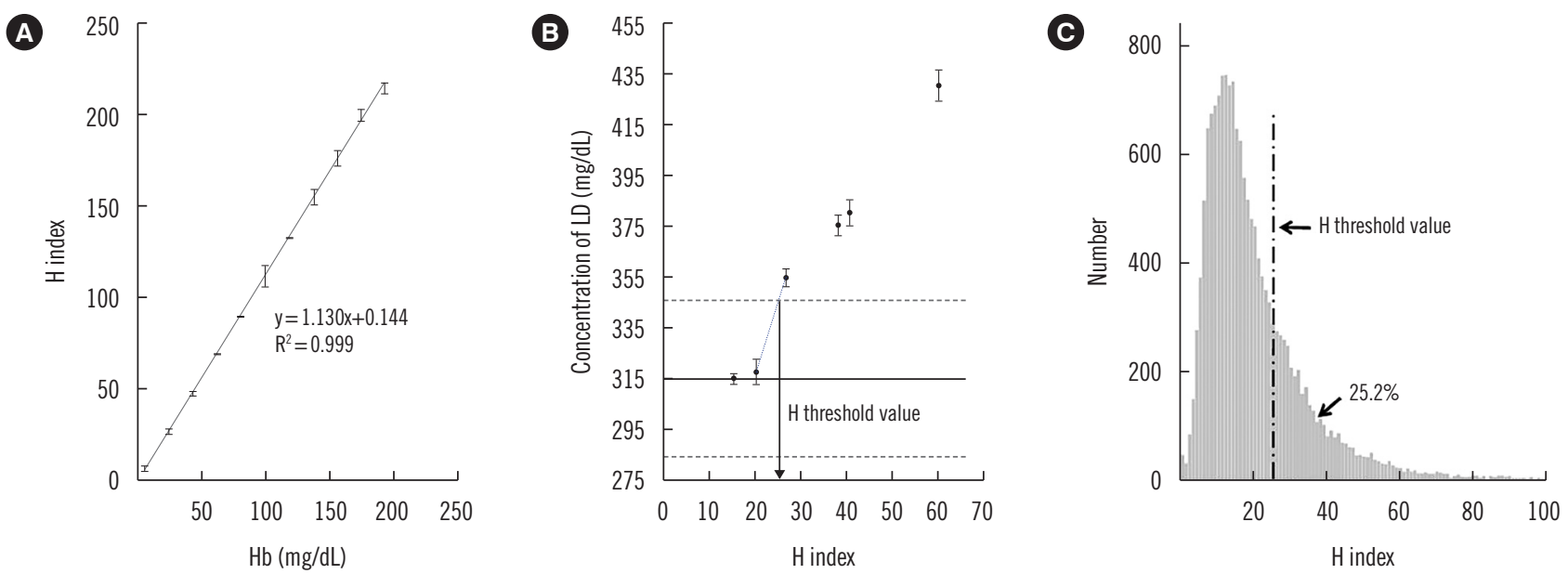

Fig. 1. Correlation between the $\mathrm{H}$ index and $\mathrm{Hb}$ concentration (A). Interference with the measurement of LD caused by increased hemolysis in a sample (B). The solid line represents the original concentration of LD, and the dotted lines denote a $10 \%$ difference from the original level. The $\mathrm{H}$ index value of 25 was expected to cause a $10 \%$ bias in the measurement. The distribution of $\mathrm{H}$ index values among all the requested routine chemistry samples in a given month $(\mathrm{C})$. The proportion of samples with an $\mathrm{H}$ index $>25$ was $25.2 \%$.

Abbreviations: $\mathrm{H}$ index, hemolysis index; LD, lactate dehydrogenase.

shaken for $0,6,12,18,24$, and $30 \mathrm{sec}$, respectively, to obtain samples with varying degrees of hemolysis. The LD concentration was measured by using the Cobas Reagent (Roche Diagnostics) on the Hitachi MODULAR Analytics system, and the $\mathrm{H}$ index value was simultaneously obtained. The LD concentration in the samples increased proportionally with the increase in the extent of hemolysis (Fig. 1B). In the LD measurement, the threshold $\mathrm{H}$ index value was defined as the level corresponding to the point at which hemolysis induces a $10 \%$ bias, which is the allowable limit of bias suggested by the manufacturer [11]. The $\mathrm{H}$ index value was estimated to be 25 (Fig. 1B), which was used in this study as the threshold $\mathrm{H}$ index resulting in significant interference. Investigation of all requested clinical chemistry samples in one month (May 2015) revealed that 25.2\% of the samples had an $\mathrm{H}$ index higher than the threshold value of 25 (Fig. 1C). This finding indicated that a substantial proportion of the samples are associated with a risk of significant analytical error.

To develop a quality improvement indicator reflecting improper acquisition and handling of samples, we compared the monthly mean $\mathrm{H}$ index and the monthly frequency of clinical samples with an $\mathrm{H}$ index over 25 by the type of ward and time period. For the comparison of $\mathrm{H}$ index values among the types of ward, we analyzed 7,813 samples requested from the Division of Gastroenterology at Hallym University Sacred Heart Hospital from January to June 2015. The samples were grouped by the type of ward of origin: ER, medical intensive care unit (MICU), GW, and OPD. The data on each group are expressed as the mean of monthly results \pm SD. The differences among the four groups were analyzed by the Kruskal-Wallis test, and the difference within each pair of groups was analyzed by the Mann-Whitney test using the SPSS Statistics software, version 21 (IBM, Chicago, IL, USA), and Microsoft Excel (Microsoft Corporation, Redmond, WA, USA). Significance was determined with a $P$ value threshold of 0.05 for a two-tailed test. The monthly mean $\mathrm{H}$ index differed significantly among the types of ward $(P=0.01)$. The monthly mean $\mathrm{H}$ indices of the samples from the ER $(36.1 \pm 2.23)$ and the MICU $(30.2 \pm 5.64)$ were significantly higher than those of the samples from the GW $(20.6 \pm 5.58, P=0.01$ and $P=0.03$, respectively) or OPD $(16.9 \pm 1.00, P=0.01$ for both; Fig. $2 A)$. There was no significant difference in the monthly mean $\mathrm{H}$ index between the $\mathrm{ER}$ and MICU and between the GW and OPD. The monthly frequency of samples with an $\mathrm{H}$ index $>25$ also differed significantly $(P=0.01)$ among the types of ward in the following order: $\mathrm{ER}, 58.6 \pm 2.27 \%$; MICU, $37.7 \pm 6.09 \%$; GW, $21.2 \pm 6.42 \%$; and OPD, $15.6 \pm 2.33 \%$. In the pairwise comparison between groups, data within each pair differed significantly $(P=0.01)$ except for the comparison between samples from the GW and OPD (Fig. 2B).

In the middle of the study period, a centrifuge in a clinical chemistry laboratory was replaced because of defective temperature control. We compared the $\mathrm{H}$ index values of 31,672 samples requested for routine chemistry tests before centrifuge replacement (4 months, from September to December 2014) to those of 53,604 samples tested after the replacement (5 months, from February to June 2015). Differences in the 
A

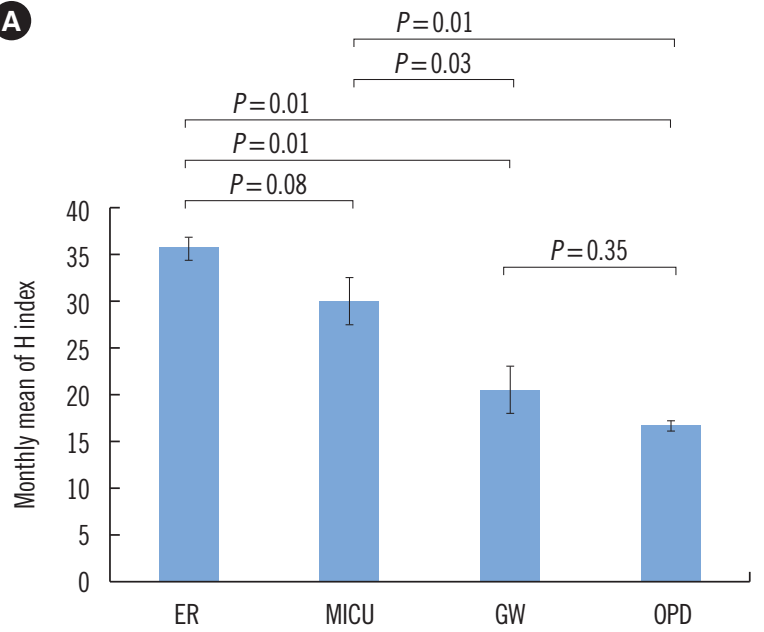

B

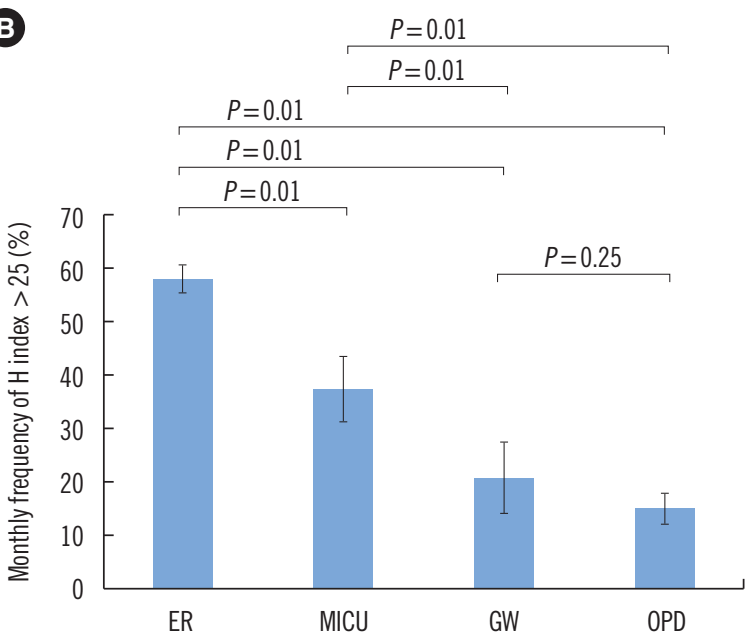

Fig. 2. The monthly mean $H$ index (A) and the monthly frequency of samples with an $H$ index over 25 (B) by the type of ward. The data are presented as the mean $\pm S D$ of monthly values during six months (from January to June 2015). The differences within each pair of groups were analyzed by the Mann-Whitney U test.

Abbreviations: $\mathrm{H}$ index, hemolysis index; ER, emergency room; MICU, medical intensive care unit; GW, general ward; OPD, outpatient department.

A

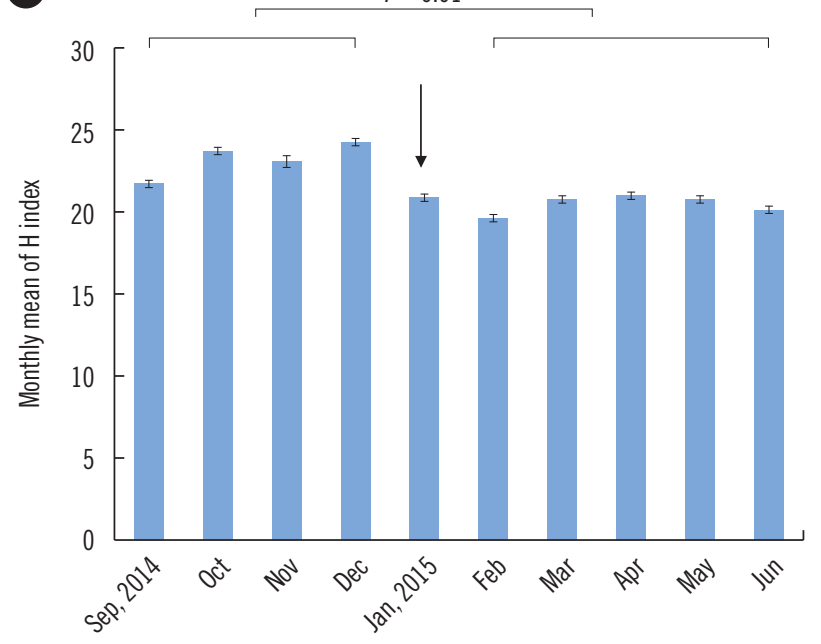

B

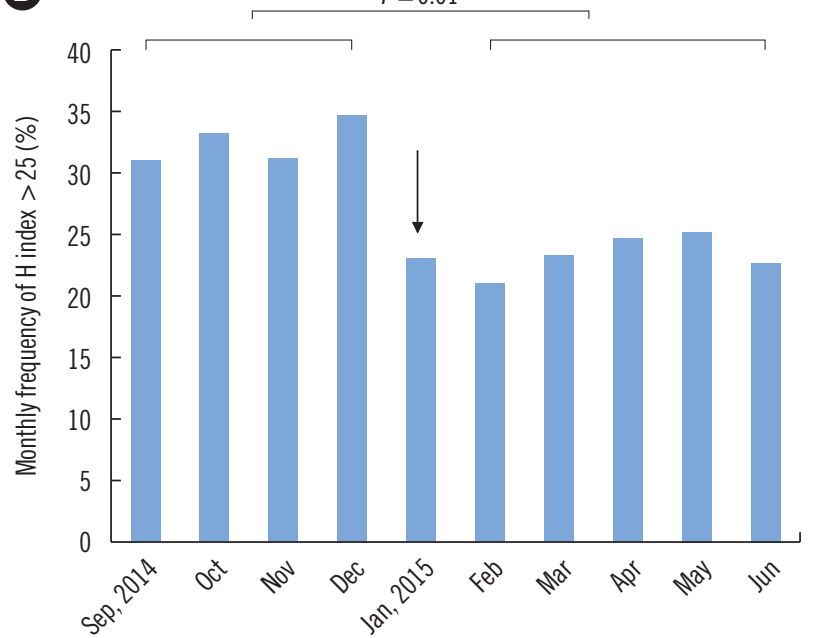

Fig. 3. The monthly mean $\mathrm{H}$ index ( $\mathrm{A}$ ) and the monthly frequency of samples with an $\mathrm{H}$ index over 25 (B) before and after replacement of a centrifuge lacking temperature control in a clinical laboratory (January 9, 2015, indicated with an arrow). Differences in the monthly mean $\mathrm{H}$ index or monthly frequency of samples with an $\mathrm{H}$ index over 25 before (from September to December 2014) and after centrifuge replacement (from February to June 2015) were analyzed by the Mann-Whitney U test.

Abbreviation: $\mathrm{H}$ index, hemolysis index.

monthly mean $\mathrm{H}$ index and monthly frequency of samples with an $\mathrm{H}$ index $>25$ were analyzed by the Mann-Whitney $\mathrm{U}$ test. The monthly mean $\mathrm{H}$ index significantly decreased after centrifuge replacement $(23.30 \pm 1.08$ vs $20.6 \pm 0.58, P=0.01$, Fig. $3 \mathrm{~A})$. The monthly frequency of the samples with an $\mathrm{H}$ index $>25$ also significantly decreased after the event $(32.6 \pm 1.78 \%$ vs $23.4 \pm 1.69 \%, P=0.01$, Fig. $3 \mathrm{~B}$ ). Both indicators clearly revealed differences in the risk of occurrence of inappropriately processed (hemolyzed) samples among the types of ward or the pathways of the sample processing, although the latter indicator seems to be more informative for the assessment of the risk.

Thus, we propose the use of the monthly mean $\mathrm{H}$ index and the monthly frequency of samples with an $\mathrm{H}$ index greater than the threshold value as quality improvement indicators. These novel indicators are easily deduced from the $\mathrm{H}$ index values of clinical samples, which are automatically obtained by means of chemical analyzers without additional reagents or costs. This method can be flexibly applied to each process pathway of 
samples in a clinical laboratory to identify the time and place of an inappropriate procedure. Broad adoption of these quality improvement indicators may help to develop effective quality improvement procedures in medical care units [12].

\section{Authors' Disclosures of Potential Conflicts of Interest}

No potential conflicts of interest relevant to this article were reported.

\section{Acknowledgments}

This study was supported by a grant (2015) from the Laboratory Medicine Foundation, Seoul, Korea.

\section{REFERENCES}

1. Blank DW, Kroll MH, Ruddel ME, Elin RJ. Hemoglobin interference from in vivo hemolysis. Clin Chem 1985;31:1566-9.

2. Van Lente F, Marchand A, Galen RS. Evaluation of a nephelometric assay for haptoglobin and its clinical usefulness. Clin Chem 1979;25:
2007-10.

3. Witte DL, Brown LF, Feld RD. Effects of bilirubin on detection of hydrogen peroxide by use of peroxidase. Clin Chem 1978;24:1778-82.

4. Spain MA and Wu AH. Bilirubin interference with determination of uric acid, cholesterol, and triglycerides in commercial peroxidase-coupled assays, and the effect of ferrocyanide. Clin Chem 1986;32:518-21.

5. Kroll MH. Evaluating interference caused by lipemia. Clin Chem 2004;50:1968-9.

6. Bornhorst JA, Roberts RF, Roberts WL. Assay-specific differences in lipemic interference in native and intralipid-supplemented samples. Clin Chem 2004;50:2197-201.

7. Aw TC and Kiechle FL. Pseudohyponatremia. Am J Emerg Med 1985;3: 236-9.

8. Lippi G, Blanckaert N, Bonini P, Green S, Kitchen S, Palicka V, et al. Haemolysis: an overview of the leading cause of unsuitable specimens in clinical laboratories. Clin Chem Lab Med 2008;46:764-72.

9. Shin DH, Kim J, Uh Y, Lee SI, Seo DM, Kim KS, et al. Development of an integrated reporting system for verifying hemolysis, icterus, and lipemia in clinical chemistry results. Ann Lab Med 2014;34:307-12.

10. Clinical Laboratory and Standards Institute. Hemolysis, icterus, and lipemia/turbidity indices as indicators of interference in clinical laboratory analysis; approved guideline, C56-A. Wayne, PA: Clinical Laboratory and Standards Institute, 2012

11. Roche Diagnostics. Serum indices: Reduction of clinical errors in laboratory medicine. http://www.rochediagnostics.cz/download/program/ Serum\%20Indices_maly.pdf. (Updated on July 2012).

12. Shahangian S and Snyder SR. Laboratory medicine quality indicators: a review of the literature. Am J Clin Pathol 2009;131:418-31. 